This item was submitted to Loughborough's Research Repository by the author.

Items in Figshare are protected by copyright, with all rights reserved, unless otherwise indicated.

\title{
An edge-based unstructured mesh discretisation in geospherical framework
}

\section{PLEASE CITE THE PUBLISHED VERSION}

http://dx.doi.org/10.1016/j.jcp.2010.03.017

\section{PUBLISHER}

(c) Elsevier

VERSION

AM (Accepted Manuscript)

\section{LICENCE}

CC BY-NC-ND 4.0

\section{REPOSITORY RECORD}

Szmelter, Joanna, and Piotr K. Smolarkiewicz. 2019. "An Edge-based Unstructured Mesh Discretisation in Geospherical Framework”. figshare. https://hdl.handle.net/2134/13382. 
This item was submitted to Loughborough's Institutional Repository (https://dspace.lboro.ac.uk/) by the author and is made available under the following Creative Commons Licence conditions.

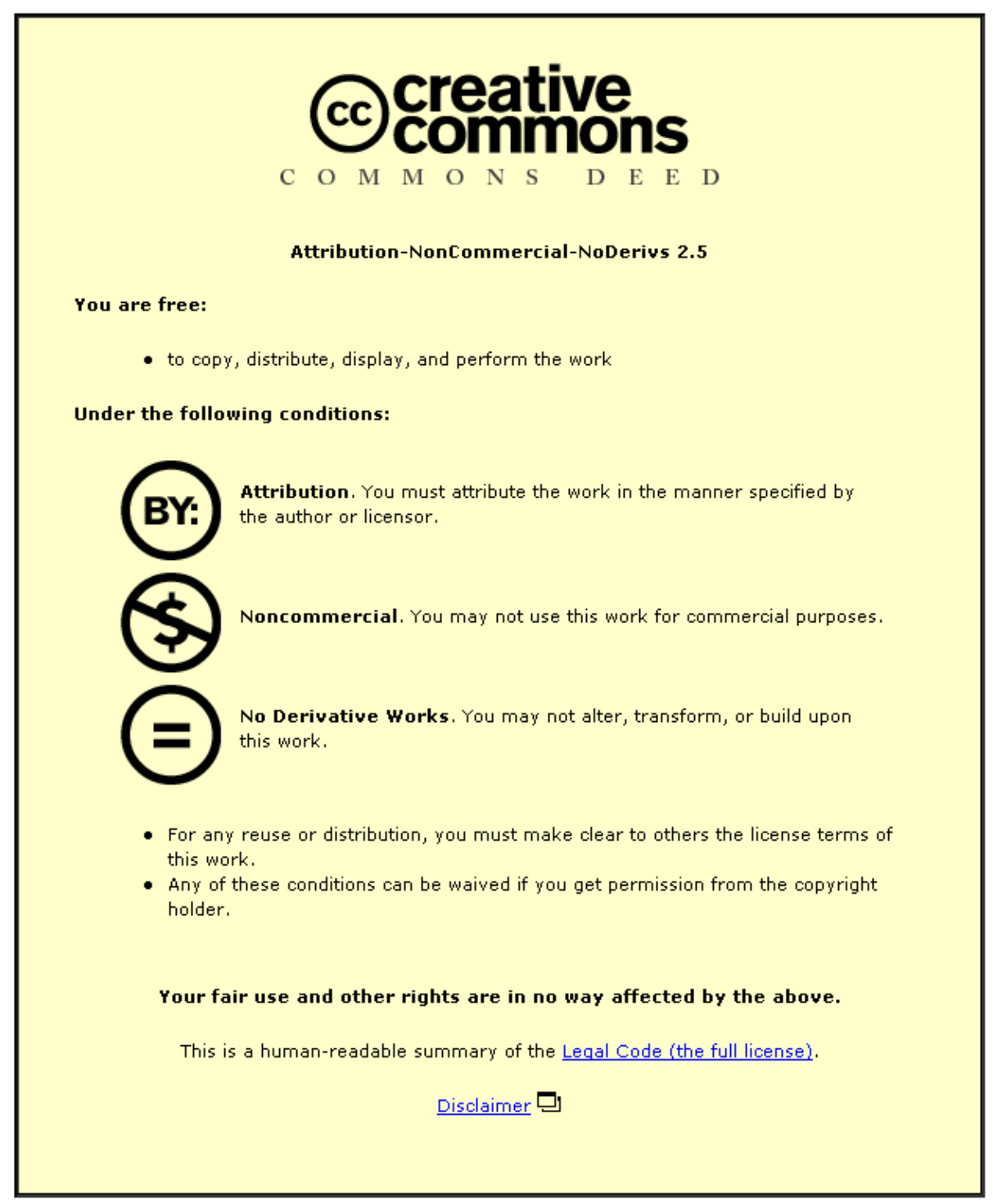

For the full text of this licence, please go to: http://creativecommons.org/licenses/by-nc-nd/2.5/ 


\title{
An Edge Based Unstructured Mesh Discretisation in Geospherical Framework
}

\author{
Joanna Szmelter ${ }^{a}$ \\ ${ }^{a}$ Loughborough University, Leicestershire LE11 3TU, UK \\ Piotr K. Smolarkiewicz b,* \\ ${ }^{\mathrm{b}}$ National Center for Atmospheric Research, Boulder, CO8030\%, USA
}

\begin{abstract}
An arbitrary finite volume approach is developed for discretising partial differential equations governing fluid flows on the sphere. Unconventionally for unstructuredmesh global models, the governing equations are cast in the anholonomic geospherical framework established in computational meteorology. The resulting discretisation retains proven properties of the geospherical formulation, while it offers the flexibility of unstructured meshes in enabling irregular spatial resolution. The latter allows for a global enhancement of the spatial resolution away from the polar regions as well as for a local mesh refinement. A class of non-oscillatory forward in time edge-based solvers is developed and applied to numerical examples of threedimensional hydrostatic flows, including shallow-water benchmarks, on a rotating sphere.
\end{abstract}

Key words: unstructured meshes, global models, nonoscillatory forward-in-time schemes, PDE on the sphere, edge-based finite volume schemes

PACS:

\section{INTRODUCTION}

Contemporary global models used for simulating atmospheric flows are predominantly based on the latitude-longitude (hereafter lat-lon, for brevity)

\footnotetext{
* Corresponding Author.

Email addresses: j.szmelter@lboro.ac.uk (Joanna Szmelter), smolar@ucar.edu (Piotr K. Smolarkiewicz ).
} 
structured grids with rigid connectivity. Such grids are far from optimal, because they preclude heterogeneous spatial resolution. In particular, the lack of the grid optimality impairs the efficiency of computations in polar regions [1]; whereas, in general, it can limit the accuracy of representing the multiphysics process interactions over a broad range of scales [2]. For structured grids and meshes relying on spherical symmetries, typically the only available mesh adaptivity technique is the point enrichment. However, the point enrichment impedes smooth mesh spacing and requires special treatments of interfaces between fine and coarse regions [3]. In contrast, a fully unstructuredmesh technology ${ }^{1}$ enables smooth variability of mesh spacing — e.g., in accord with flow field gradient anywhere in the computational domain - thus benefiting adaptivity techniques and providing means for minimizing truncation errors via the optimal data-point distribution. Unstructured meshes have proven record of accommodating efficiently a wide range of scales, intermittent distribution of forcing regions, and complex geometry; see [7-9] for illustrations.

The realisation of limitations of structured lat-lon grids and attraction of flexible meshing for flows on a sphere has stimulated research for over four decades; early contributions and their review include [10-13]. The early works explored a range of approaches, including hexagonal-icosaheadral, triangular, cubic and reduced grids or polar cups. The primary goal was to circumvent the CFL limitations via mesh coarsening in the polar regions, while maintaining uniform accuracy of discretisation everywhere in the mesh. More recent developments aim also at improved resolution away from the poles and explore mesh flexibility required for the effective mesh adaptivity. Notwithstanding the efforts, except for a few numerical weather prediction (NWP) models on icosahedral grids (cf. [14] and references therein), thus far there seem to be no sole alternative to the classical lat-lon formulation embraced widely in operational NWP and climate studies [15]. The quest for an alternative improving on limitations of the structured lat-lon grid continues, as reviewed recently in $[1,16,17]$. Up to date, most studies of global modelling using unstructuredmesh technology have concentrated on the solution of the shallow water equations using spherical polygonal, usually triangular, meshes. Majority of them retained some elements of the icosahedron approach [18], either by taking advantage of its straightforward procedures for mesh generation, metric properties, or regular data structure. Concomitantly, the equations of motion have been formulated using two distinct modi operandi. One approach operates in a global rotating Cartesian framework with the origin in the centre of the Earth,

1 A mesh, consisting of either irregular or regular elements, is referred to as unstructured if the discretisation associated with it does not rely on a systematic principle for identification of neighbouring points; examples of unstructured data arrangements include flexible element and edge-based connectivity, in contrast to structured data arrangements such as "i,j,k" indexing or binary trees [4,5]. 
e.g. [19,2]. It circumvents theoretical complexities of differential manifolds (cf. Chpt. XIV in [20]) by evaluating a flow on the sphere's surface directly as a three-dimensional flow in an Euclidean space. Another approach follows the formalism of calculus on manifolds, while employing local coordinate mappings with origins representing individual computational cells on the sphere's surface; see [16] for a comprehensive discussion, list of references and example of numerical realization.

Here, we report on the development of a different approach. In essence, we start with the governing equations of motion formulated in the classical latlon framework, section 7.2 in [21], and circumvent limitations of the framework by discretising the equations using an unstructured-mesh technology. It might be argued that this is still akin to the second modus operandi, as the classical framework does adhere to the principles of differential geometry. Yet its atlas consists of only three charts, with the primary chart covering nearly the entire surface of the sphere and the remaining two degenerated to the special boundary conditions for differentiating dependent variables in the vicinity of the poles. Nevertheless, the aim of our approach is distinct, as its goal is to retain the benefits of the classical formulation - common in theoretical geo/astro physics — while alleviating its shortcomings by manipulating the inhomogeneity and anisotropy of discretisation admitted by unstructured meshes. This is diametrically different from covering the spherical surface with multitude of charts to assure the uniformity of distretisation.

There are multiple benefits in adopting the geospherical coordinates approach, and most global atmospheric models employ it. For lat-lon grids the benefits of straightforward grid specification, analytic evaluation of geometrical metrics, and maturity of flow solvers relying on index data structure and grid orthogonality make the geospherical framework a natural choice. However, other proven properties of the classical geospherical formulation are not grid specific and can be retained on more flexible unstructured meshes. The key one of such desirable properties is the physicality of the velocity vector, [21], with zonal and meridional wind components directly measurable in local Cartesian coordinate systems. This is important for the conservativity and the accuracy of a numerical model formulation (section 3.3 in [22]) emphasizing mean/climatic circulations of planetary and stellar systems. Another beneficial property of the geospherical framework is an exact analytic representation of the spherical surface. This aspect is offered also by schemes utilising icosahedral properties but at the price of limited mesh flexibility and complexity in constructing high-order spatial operators [16]. Some finite-element global models provide flexible meshing by using various forms of isoparametric elements (e.g., based on polynomial mappings [23]) for sphere discretisation. In effect, the accuracy of representing the geometry of a spherical surface depends on the elements used. Conversely, inaccurate representation of spherical surface can introduce significant errors in solution of the governing PDEs. Consider that if the cur- 
vature of triangular elements is neglected by covering the Earth with flat tiles [19], the resulting errors in the radial displacement of an element (and arguably, of the isentropes aloft) are $\sim r(\delta \alpha)^{2} / 8$ where $(\delta \alpha$ and $r$ denote the angular resolution and sphere's radius, respectively) - i.e, insignificant for elements with angular size of 0.1 degree, but comparable to $200 \mathrm{~m}$ tall mountains at a $1^{\circ}$ resolution. Yet another benefit of formulating the problem in the geospherical framework is that it simplifies the unstructured-mesh generation. Notably, the required computational meshes are generated for a simple geometry of the primary chart. In consequence, the mesh generation (and implementation of mesh manipulation techniques used in adaptivity) are much easier than for the approaches that require generating flexible meshes directly on a sphere embedded in an Euclidean space. For example, in two-dimensional (2D) shallow water and three-dimensional (3D) hydrostatic models of the type illustrated in this paper, flexible meshes are generated for a rectangle - in the same spirit as the regular lat-lon grid. In general, 3D meshes in the geospherical framework are generated for a simple hexahedron; whereas, a generation of $3 \mathrm{D}$ prismatic meshes, commonly used in atmospheric global models is straightforward.

The generalisation of geospherical framework to arbitrary unstructured meshes offers an efficient numerical development path for extending mature methods operating on lat-lon grids to flexible meshes. In particular, the presented approach builds on the methodologies developed for an established structuredgrid computational model EULAG for simulating thermo-fluid flows across a wide range of scales and physical scenarios; see [24] for a recent review. EULAG has a proven record in fluid dynamics of rotating stratified flows and in diverse areas of atmospheric applications. Its numerical concepts form a particularly convenient base for advancing the proposed generalisation to flexible unstructured-mesh model. Especially relevant is the capability of EULAG's algorithms to accommodate abruptly changing control volumes (due to the underlying mathematical and numerical model formulation in generalised, time dependent curvilinear coordinates), demonstrated with diverse tests across a range of scales and problems from mesoscale gravity wave dynamics to idealised terrestrial climate $[25,26]$.

The first necessary step towards enabling the generalisation of EULAG's methodologies to hybrid unstructured meshes was the derivation of MPDATA (multidimensional positive definite advection transport algorithm [27], a key feature of EULAG) for an edge-based data structure [28,29]. Further essential steps included developments of a class of edge based non-oscillatory forwardin-time solvers for compressible fluid equations [30,31]. Moreover, the potential for implementation of mesh adaptivity to the edge-based MPDATA methodology was explored in [6] where an explicit, analytical form of the error estimator naturally arising from MPDATA was used in combination with mesh movement, remeshing and mesh enrichment strategies. Comparisons reported for 
these edge based developments show that the unstructured mesh codes retain proven properties of the standard EULAG and embody structured grids as a special case.

In the following section we introduce an abstract form of governing equations written in geospherical framework. Section 3 presents features of the analytical and numerical aspects of the unstructured-mesh algorithms developed for global flows. Section 4 substantiates the theoretical and numerical formulation of the approach with selected benchmark calculations for the shallow water equations. Section 5 illustrates the potential of the unstructured-mesh discrtisation in geospherical framework for simulating geophysical flows across scales. It employs examples of (effectively) meso-scale orographic 3D flows at a range of Froude numbers, using mesh refinement on reduced planets [32]. Remarks in section 6 conclude the paper.

\section{GEOSPHERICAL FRAMEWORK}

The notation and terminology adopted throughout this paper bridge the tensorial formalism of the presentation in [25] with the nomenclature traditional in global-scale atmospheric applications, stemming from the use of orthogonal coordinates $[33,34]$. In [25] the integrations of PDEs governing atmospheric dynamics were generalised for time-dependent non-orthogonal curvilinear coordinates, to enable dynamic grid adaptivity by means of continuous mappings. The adopted notation aims at future development of a unified framework for combining continuous mappings with an unstructured-mesh discretisation.

We consider finite-volume approximations for an inhomogeneous archetype PDE representing a conservative form of an evolutionary problem for a scalar variable $\psi$ advected with a fluid flow on the sphere

$$
\frac{\partial G \psi}{\partial t}+\nabla \cdot\left(G \mathbf{v}^{*} \psi\right)=G R
$$

From the perspective of numerical methods, such an abstract PDE underlies a range of applications from elementary advective transport of the density of a passive tracer to elaborate systems of PDE describing complex dynamics of weather and climate $[24,31]$. In (1) the Jacobian $G \equiv\left|g_{p q}\right|^{1 / 2}$ is defined in terms of the metric tensor $g_{p q}$ of the spherical coordinate system $\mathbf{x}=\left(x^{1}, x^{2}\right) \equiv(x, y) \equiv(\lambda, \varphi)$ with the metric form $d s^{2}=g_{p q} d x^{p} d x^{q}=$ $g_{11} d x^{1} d x^{1}+g_{22} d x^{2} d x^{2} \equiv\left(h_{x} d \lambda\right)^{2}+\left(h_{y} d \varphi\right)^{2}$, where $h_{x}=\sqrt{g_{11}}=r \cos \varphi$, $h_{y}=\sqrt{g_{22}}=r$ with $\lambda, \varphi$ and $r$ denoting, respectively, the longitude and latitude angles and the sphere's radius. Consequently, $G=h_{x} h_{y}, \mathbf{v}^{*}=\dot{\mathbf{x}}$ denotes the contravariant velocity, and $\nabla=(\partial / \partial x, \partial / \partial y) \equiv(\partial / \partial \lambda, \partial / \partial \varphi)$. Whenever 
$R$ refers to the right-hand-side of a momentum equation, it accounts for pressure gradient terms as well as Coriolis and metric forces in a form familiar from meteorological applications [33,34]. An elementary example illustrating the symbolic form in (1) is the set of shallow water equations

$$
\begin{aligned}
\frac{\partial G \mathcal{D}}{\partial t}+\nabla \cdot\left(G \mathbf{v}^{*} \mathcal{D}\right) & =0 \\
\frac{\partial G Q_{x}}{\partial t}+\nabla \cdot\left(G \mathbf{v}^{*} Q_{x}\right) & =G\left(-\frac{\mathrm{g}}{h_{x}} \mathcal{D} \frac{\partial H}{\partial x}+f Q_{y}-\frac{1}{G \mathcal{D}} \frac{\partial h_{x}}{\partial y} Q_{x} Q_{y}\right), \\
\frac{\partial G Q_{y}}{\partial t}+\nabla \cdot\left(G \mathbf{v}^{*} Q_{y}\right) & =G\left(-\frac{\mathrm{g}}{h_{y}} \mathcal{D} \frac{\partial H}{\partial y}-f Q_{x}+\frac{1}{G \mathcal{D}} \frac{\partial h_{x}}{\partial y} Q_{x}^{2}\right),
\end{aligned}
$$

where $\mathcal{D}$ and $H$ denote, respectively, the depth of the shallow water and the height of its surface (in the absence of orography/bathymetry, $H \equiv \mathcal{D}$ ), g is the gravitational acceleration, and $f=f_{0} \sin \varphi$ is the Coriolis parameter. The momentum vector $\mathbf{Q}=\mathcal{D} \mathbf{v}$, where $\mathbf{v}$ denotes a physical velocity (with dimensions of length/time), related to the contravariant velocity via

$$
\left(v_{x}, v_{y}\right)=\left(h_{x} v_{x}^{*}, h_{y} v_{y}^{*}\right) \equiv\left(h_{x} \dot{\lambda}, h_{y} \dot{\varphi}\right)
$$

Notably, when accounting for the mass continuity equation, the momentum equation in (2) is mathematically equivalent to the Newtonian-law form for the velocity evolution.

$$
\mathcal{D} \frac{d \mathbf{v}}{d t} \equiv \mathcal{D}\left(\frac{\partial}{\partial t}+\mathbf{v}^{*} \cdot \nabla\right) \mathbf{v}=\mathbf{R}
$$

where $\mathbf{R}=\left(R_{x}, R_{y}\right)$ is a shorthand for the forces in the parenthetic terms on the rhs of equations for $\left(Q_{x}, Q_{y}\right)$ in $(2)$.

\section{NUMERICAL APPROXIMATIONS}

\subsection{Non-oscillatory forward-in-time algorithm}

For integrating PDEs governing fluid motion on the sphere, we employ the nonoscillatory forward-in-time (NFT) template algorithm generalised to the edgebased data structure [31]. Here, we extend it to the geospherical framework. A set of conservative PDEs (1) can be written in a vector form as

$$
\frac{\partial G \boldsymbol{\Phi}}{\partial t}+\nabla \cdot(\mathbf{V} \boldsymbol{\Phi})=G \mathbf{R}
$$


where $\boldsymbol{\Phi}$ is a vector of dependent variables - $\left(\mathcal{D}, Q_{x}, Q_{y}\right)$ in the case of $(2)-$ and $\mathbf{R}$ is the vector of the associated rhs forcings. Furthermore, $\mathbf{V}=G \mathbf{v}^{*}$ is the advective velocity - termed as such, for the role it plays in advection schemes; cf. section 4.1 of [27]. The corresponding NFT algorithm approximates the space-time control volume integral of (5) directly (i.e., without splitting) to the second order ${ }^{2}$ as

$$
\begin{aligned}
\forall_{i, n} \quad \boldsymbol{\Phi}_{i}^{n+1} & =\mathcal{A}_{i}\left(\boldsymbol{\Phi}^{n}+0.5 \delta t \mathbf{R}^{n}, \mathbf{V}^{n+1 / 2}, G\right)+0.5 \delta t \mathbf{R}_{i}^{n+1} \\
& \equiv \widehat{\boldsymbol{\Phi}}_{i}+0.5 \delta t \mathbf{R}_{i}^{n+1} .
\end{aligned}
$$

where $n$ and $i$ have usual meaning of the temporal and spatial position, and $\mathcal{A}$ is a shorthand for the edge based MPDATA [28]. Following the experience with structured-grid MPDATA schemes for continuous mappings — see [27] and references therein - the reduction of the Jacobian $G$ in (6) is accounted for within the discrete transport operator, the technical aspects of which will be explained shortly. The $\mathbf{V}^{n+1 / 2}$ argument of $\mathcal{A}$ denotes a $\mathcal{O}\left(\delta t^{2}\right)$ estimate of $G \mathbf{v}^{*}$ at $t+0.5 \delta t$; whereas $\mathbf{R}^{n+1}$ is a second-order-accurate finite-volume representation of $\mathbf{R}$. For the overall second-order accuracy of (6), it would suffice to consider only an $\mathcal{O}(\delta t)^{2}$ representation of $\mathbf{R}$, yet with $\mathbf{R}^{n+1}=\mathbf{R}(t+$ $\delta t)+\mathcal{O}\left(\delta t^{3}\right)$ the algorithm (6) admits schemes for wave propagation with zero amplitude error [35].

Implementing the template (6) requires two specifications: i) a first-order estimate of the advecting velocity $\mathbf{V}$ at $t^{n+1 / 2}$; and ii) a second-order estimate of the rhs $\mathbf{R}$ at $t^{n+1}$. For $\mathbf{V}^{n+1 / 2}$ one can use either a linear extrapolation, or a first-order solution to the governing system. It has been shown in [35], that integrating the evolutionary form (4) - rather than the mathematically equivalent conservation form (1) — provides means of stabilisation for elastic problems on co-located grids. Here we use this option exclusively, employing over half $\delta t$ the centered-in-space forward-in-time Euler scheme for each component of the physical velocity vector $\mathbf{v}$

$$
\forall_{i, n} \quad \widetilde{\mathbf{v}}_{i}^{n+1 / 2}=\mathbf{v}_{i}^{n}-\left.0.5 \delta t \mathbf{v}^{*}\right|_{i} ^{n} \cdot \nabla_{i} \mathbf{v}+0.5 \delta t \check{\mathbf{R}}_{i}^{n}
$$

where $\nabla_{i}$ denotes a centered finite-volume approximation of the gradient operator at the $i$ th node, and $\check{\mathbf{R}}$ symbolizes the specific counterpart of $\mathbf{R}$ (e.g. $\mathbf{R} / \mathcal{D}$ in the case of $(2))$. The advective velocity in (6) is computed readily in the sequence $\left.\widetilde{\mathbf{v}}_{i}^{n+1 / 2} \rightarrow \mathbf{v}^{*}\right|_{i} ^{n+1 / 2}$ and $\left.\mathbf{v}^{*}\right|_{i} ^{n+1 / 2} \rightarrow\left(G \mathbf{v}^{*}\right)_{i}^{n+1 / 2}=\mathbf{V}_{i}^{n+1 / 2}$ by means of local transformations. In the case of (2) with the transformation (3), $\mathbf{V}_{i}^{n+1 / 2}=\left(h_{y} \widetilde{v}_{x}, h_{x} \widetilde{v}_{y}\right)_{i}^{n+1 / 2}$.

2 The NFT algorithm is congruent with the trapezoidal trajectory integral of the evolutionary form of (5); see section 2.2 in [31] for a recent discussion. 
In general, a provision of an $\mathcal{O}\left(\delta t^{3}\right)$ estimate of $R^{n+1}$ in (6) is problem dependent; see [31] for a range of examples. For all computations reported in this paper, $R \equiv 0$ in the mass continuity equation; advancing its solution first provides explicitly the pressure gradient force in $\mathbf{R}$ for momenta; hereafter $\mathbf{R e}$. However, the Coriolis and metric forces depend on momenta and are, therefore, implicit; hereafter Ri. Rewriting the rhs forcing as the sum of explicit and implicit contributions $\mathbf{R}=\mathbf{R e}+\mathbf{R i}$, leads to a refined form of (6)

$$
\forall_{i, n} \quad \mathbf{Q}_{i}^{n+1}=\widehat{\widehat{\mathbf{Q}}}_{i}+0.5 \delta t \mathbf{R i}_{i}^{n+1}
$$

where $\widehat{\widehat{\mathbf{Q}}} \equiv \widehat{\mathbf{Q}}+0.5 \delta t \mathbf{R e}^{n+1}$, with $\widehat{\mathbf{Q}}$ defined by the identity in (6). In shallowwater examples discussed later in the paper, (8) is iterated as

$$
\forall_{i, n} \quad \mathbf{Q}_{i}^{n+1, \mu}=\widehat{\widehat{\mathbf{Q}}}_{i}+0.5 \delta t \mathbf{R i}\left(\mathcal{D}_{i}^{n+1}, \mathbf{Q}_{i}^{n+1, \mu-1}\right)
$$

where $\mu=1, . ., m$ numbers successive iterations, and the first guess $\mathbf{R i}^{n+1,0}$ is either a first-order predictor or, simply, $\mathbf{R} \mathbf{i}^{n+1,0}=\mathbf{R i}^{n}$. In the $3 \mathrm{D}$ examples extending the shallow-water equations to rotating stratified flows, the $\mathbf{R i}$ is further decomposed into the Coriolis and metric terms $\mathbf{R i}=\mathbf{C Q}+\mathbf{M}(\mathcal{D}, \mathbf{Q})$, respectively, linear and nonlinear in $\mathbf{Q}$; here $\mathbf{C}$ refers to the skew-symmetric matrix of the Coriolis-force coefficients. In consequence, only the metric terms are iterated, whereas the Coriolis terms are inverted algebraically according to

$$
\forall_{i, n} \quad \mathbf{Q}_{i}^{n+1, \mu}=\widetilde{\widetilde{\mathbf{Q}}}_{i}+0.5 \delta t[\mathbf{I}-\mathbf{C}]^{-1} \mathbf{M}\left(\mathcal{D}_{i}^{n+1}, \mathbf{Q}_{i}^{n+1, \mu-1}\right)
$$

where $\widetilde{\widetilde{\mathbf{Q}}}=[\mathbf{I}-\mathbf{C}]^{-1} \widehat{\widehat{\mathbf{Q}}}$, and $\mathbf{I}$ is the identity matrix. Because in all problems considered in this paper the time-scales associated with the Coriolis and metric forces are much longer than the time-scale of advection and gravity waves, the $\delta t$ required for the computational stability also warrants rapid convergence of iterations in (9) and (10). Depending on the initial guess, at most two iterations suffice for providing an $\mathcal{O}\left(\delta t^{3}\right)$ estimate of the entire $\mathbf{R}^{n+1}$ for momenta. Notably, the computationally intensive explicit part $\widehat{\boldsymbol{\Phi}}$ of (6) is evaluated only once per time step; cf. [34,31] for further discussion. Moreover, because (6) assumes a co-located mesh, the iterative operations involve only nodal values.

\subsection{Edge-based finite-volume discretisation}

For flow problems considered in this paper, the spatial discretisation assumed in the template algorithm (6) uses the 2D edge-based median-dual finite vol- 
ume approach [28]. This approach allows to circumvent theoretical complexity of tensorial formulation, by integrating the generic physical form of the governing PDE over arbitrarily-shaped cells on an arbitrary manifold, and it lends itself well to various mesh adaptivity techniques [28,6]. A schematic of the edge-based data structure for an arbitrary hybrid mesh on a 2D plane is shown in Fig. 1. The median-dual finite-volume approach constructs the control volume associated with the vertex $i$ by joining the centers of polygonal mesh cells encompassing the vertex $i$ and midpoints of edges originating in the vertex $i$, Fig. 1. The approach is readily applicable to 3D hybrid meshes [28].

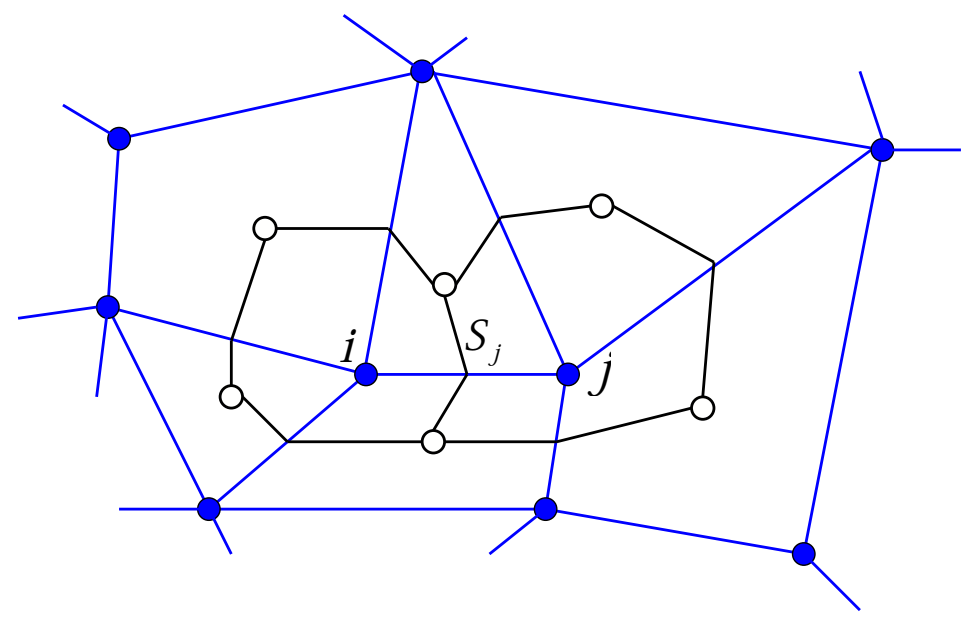

Fig. 1. The edge-based, median-dual approach in 2D. The edge connecting vertices $i$ and $j$ pierces the face $S_{j}$ shared by $2 \mathrm{D}$ computational (dual) cells surrounding vertexes $i$ and $j$. Open circles represent centers of the polygonal mesh cells.

Having defined the mesh in planar geometry, ${ }^{3}$ all geometric elements such as cell volume, cell face area, and normals are evaluated from elementary vector calculus. Hereafter, $\mathbf{S}_{j} \equiv S_{j} \mathbf{n}_{j}$ symbolizes the oriented surface element with $\mathbf{n}_{j}$ denoting the normal; $\mathcal{V}_{i}^{\bullet}$ is the planar area of the cell containing vertex $i$. In the geospherical framework all metric aspects are accounted for analytically. Thus, the governing set of geospherical PDEs (5) can be viewed as a set of modified planar PDEs. In particular, the transformations defining the advective velocity in terms of the contravariant and physical velocities, and the definition of the finite-volume cell area on the surface of the sphere $\mathcal{V}_{i}=G_{i} \mathcal{V}_{i}^{\bullet}$ suffice for implementing the MPDATA operator $\mathcal{A}$ in (6).

Let $\Psi=\Phi^{n}+0.5 \delta t R^{n}$ in (6). Technically, MPDATA consists of a series of the first-order upwind (alias donor-cell) steps, with the first step providing firsorder-accurate solution, and subsequent steps compensating the truncation

3 This immediate benefit of the geospherical framework makes the task of mesh generation particularly straightforward compared to procedures required by methods using meshes defined directly on a sphere. 
error of the preceding step while preserving the sign of the transported variable $\Psi$. Employing the Gauss divergence theorem in the edge-based finite-volume framework [28], each upwind step can be written as

$$
\Psi_{i}^{n+1}=\Psi_{i}^{n}-\frac{\delta t}{\mathcal{V}_{i}} \sum_{j=1}^{l(i)} F_{j}^{\perp} S_{j}
$$

The fluxes $F j^{\perp}$ are interpreted as the mean normal fluxes through the cell face $S_{j}$ averaged over the temporal increment $\delta t$. They assume the functional dependence on the cell-volume averaged field $\Psi$ and the normal advective velocity $V^{\perp}$ at the face $S_{j}$, in a form

$$
F_{j}^{\perp}\left(\Psi_{i}, \Psi_{j}, V_{j}^{\perp}\right)=\left[V_{j}^{\perp}\right]^{+} \Psi_{i}+\left[V_{j}^{\perp}\right]^{-} \Psi_{j},
$$

with

$$
[V]^{+} \equiv 0.5(V+|V|), \quad[V]^{-} \equiv 0.5(V-|V|)
$$

The nonnegative/nonpositive parts of $V_{j}^{\perp}$ always coincide with outflow/inflow from the $i$ th cell. The summation in $(11)$ is over all $l(i)$ edges connecting vertex $i$ with its immediate neighbors $j$, and $S_{j}$ refers both to the cell face pierced by the $j$ th edge and to its oriented surface area; see Fig. 1. With this notation, the entire MPDATA procedure can be written in a compact functional form as

$$
\Psi_{i}^{(k)}=\Psi_{i}^{(k-1)}-\frac{\delta t}{\mathcal{V}_{i}} \sum_{j=1}^{l(i)} F_{j}^{\perp}\left(\Psi_{i}^{(k-1)}, \Psi_{j}^{(k-1)}, V_{j}^{\perp,(k)}\right) S_{j}
$$

with $k=1, . ., I O R D$ such that

$$
\begin{gathered}
\Psi^{(0)} \equiv \Psi^{n} ; \Psi^{(I O R D)} \equiv \Psi^{n+1} \\
V^{\perp,(k+1)}=V^{\perp}\left(\mathbf{V}^{(k)}, \Psi^{(k)}, \nabla \Psi^{(k)}\right) \quad ;\left.\quad V_{j}^{\perp,(1)} \equiv V^{\perp}\right|_{j} ^{n+1 / 2} .
\end{gathered}
$$

In (14), the first iteration uses for arguments of $F_{j}^{\perp}$ the transported field values from the preceding time step and an $\mathcal{O}\left(\delta t^{2}\right)$ estimate of the velocity at $t^{n+1 / 2}$. In context of the governing equations (2),

$$
\left.V^{\perp}\right|_{j} ^{n+1 / 2} S_{j}=\left(\delta y \frac{\left(h_{y} \widetilde{v}_{x}\right)_{i}+\left(h_{y} \widetilde{v}_{x}\right)_{j}}{2}-\delta x \frac{\left(h_{x} \widetilde{v}_{y}\right)_{i}+\left(h_{x} \widetilde{v}_{y}\right)_{j}}{2}\right)^{n+1 / 2}
$$

where $\delta x=x_{j}-x_{i}$ and $\delta y=y_{j}-y_{i}$ are the zonal and meridional angular increments, and $\widetilde{v}_{x}$ and $\widetilde{v}_{y}$ are the components of the physical velocity predictor (7). In subsequent iterations in (14), the compensating antidiffusive 
Table 1

Scalar advection on an irregular triangular mesh (cf. Fig. 7); solid-body rotation past the poles. The two rows list, respectively: a) spatial (and temporal) resolution relative to a reference mesh with $128 \times 64$ nodes; and b) $L_{2}$ norm of the MPDATA solution error.

\begin{tabular}{ccccc}
\hline \hline$\delta \xi / \delta \xi_{o}$ & 6 & 3 & 1.5 & 0.75 \\
$L_{2}$ & 0.01253 & 0.00428 & 0.00111 & 0.00028 \\
\hline \hline
\end{tabular}

velocity is defined by the leading truncation error of the preceding upwind step as $V^{\perp,(k+1)} \equiv-(\text { Error } / \psi)^{(k)}$; namely,

$$
\begin{aligned}
V_{j}^{\perp,(k+1)} & =\left|V_{j}^{\perp,(k)}\right| \frac{\left|\Psi_{j}^{k}\right|-\left|\Psi_{i}^{k}\right|}{\left|\Psi_{j}^{k}\right|+\left|\Psi_{i}^{k}\right|+\varepsilon} \\
& -\frac{\delta t}{2} V_{j}^{\perp,(k)}\left(\mathbf{V}^{(k)} \cdot \frac{\nabla\left|\Psi^{k}\right|}{\left|\Psi^{k}\right|}+\nabla \cdot \mathbf{V}^{(k)}\right)_{S_{j}}
\end{aligned}
$$

where $\mathbf{V}^{(k)}=G \mathbf{v}^{*(k)}$ and $\varepsilon$ denotes a small constant, e.g. $10^{-10}$, to assure that the denominator does not vanish where $\Psi_{j}^{(k)}=\Psi_{i}^{(k)}=0$. In practice, one corrective iteration suffices for recovering the second-order accuracy of time-space centered schemes. The latter is highlighted in table 1 that lists the solution errors in function of the unstructured-mesh characteristic spacing, for scalar advection of a cosine-bell tracer profile in a rotational flow past the poles with constant angular velocity - a test case that we adopted from [36].

The remaining implementation details of (16) follow the planar formulation [6]. Applying the Gauss theorem to an augmented vector field $\Psi \mathbf{e}^{I}$, with $\mathbf{e}^{I}$ denoting a unit vector in the $I$ th direction, the partial derivatives are evaluated as

$$
\left(\frac{\partial \Psi}{\partial x^{I}}\right)_{j}=\frac{1}{\overline{\mathcal{V}}_{j}}\left(\sum_{m=1}^{l(i)} \bar{\Psi}^{i, m} S_{m}^{I}+\sum_{m^{\prime}=1}^{l(j)} \bar{\Psi}^{j, m^{\prime}} S_{m^{\prime}}^{I}\right), \overline{\mathcal{V}}_{j} \equiv \mathcal{V}_{i}+\mathcal{V}_{j}
$$

where, $\Psi \equiv\left|\Psi^{(k)}\right|, \bar{\Psi}^{i, m} \equiv 0.5\left(\Psi_{i}+\Psi_{m}\right)$, and $S_{m}^{I}$ denotes the $I$ th area component of the oriented surface element at the $m$ th edge. The associated " $\bar{~} "$ denominator in the second term on the rhs of (16) is evaluated as a surface-area weighted average from the same control volume

$$
\bar{\Psi}_{j}=\frac{1}{\overline{\mathcal{S}}_{j}}\left(\sum_{m=1}^{l(i)} \bar{\Psi}^{i, m}\left|S_{m}^{I}\right|+\sum_{m^{\prime}=1}^{l(j)} \bar{\Psi}^{j, m^{\prime}}\left|S_{m^{\prime}}^{I}\right|+\varepsilon\right)
$$




$$
\overline{\mathcal{S}}_{j} \equiv \quad \sum_{m=1}^{l(i)}\left|S_{m}^{I}\right|+\sum_{m^{\prime}=1}^{l(j)}\left|S_{m^{\prime}}^{I}\right| .
$$

In the geospherical framework implementation of the forcing terms $R^{n}$ in (8) we evaluate gradients according to:

$$
\begin{aligned}
& \left(\frac{\partial H}{\partial x}\right)_{i}=\frac{h_{y_{i}}}{\overline{\mathcal{V}}_{i}}\left(\sum_{m=1}^{l(i)} H^{i, m} S_{m}^{x}\right) ; \\
& \left(\frac{\partial H}{\partial y}\right)_{i}=\frac{h_{x i}}{\overline{\mathcal{V}}_{i}}\left(\sum_{m=1}^{l(i)} H^{i, m} S_{m}^{y}\right) ;
\end{aligned}
$$

Recall that the spherical area of the cell, already contains the Jacobian $h_{x} h_{y}$.

\subsection{Periodic and polar boundaries}
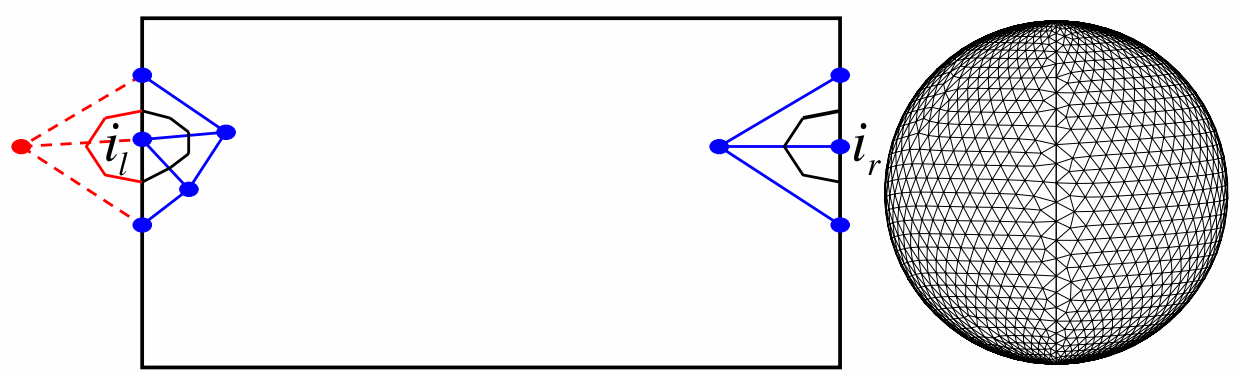

Fig. 2. Periodic boundaries: (left)a finite volumes for periodic node; (right) coarse computational mesh showing periodic nodes seen in the physical space on a sphere

For the implementation of periodic boundary conditions in $x$-direction, consider a split dual cell shown in Fig. 2. The indices $i_{l}$ and $i_{r}$ mark computational points with matching $y$ coordinate, respectively, on the left and right boundaries of the domain. The finite volume integration of (11) for the points $i_{l}$ and $i_{r}$ becomes

$$
\psi_{i_{r}}^{n+1}=\psi_{i_{l}}^{n+1}=\psi_{i_{l}}^{n}-\frac{\delta t}{\mathcal{V}_{i_{l}}+\mathcal{V}_{i_{r}}}\left(\sum_{j=1}^{l\left(i_{l}\right)} F_{j}^{\perp} S_{j}+\sum_{j=1}^{l\left(i_{r}\right)} F_{j}^{\perp} S_{j}\right),
$$

where $\mathcal{V}_{i_{l}}$ and $\mathcal{V}_{i_{r}}$ are the left and right complementary parts of the total control volume. The evaluation of derivatives in (17) and (19) follows the same principle, because it employs the Gauss divergence theorem analogously to the accumulation of fluxes in (20). 
Figure 2 highlights a particular case where for every node on the left boundary there exists a matching node on the right boundary with the coinciding coordinate $y$. For unstructured meshes this is not generally the case and we enforce it during mesh generation. Specifically, if $\left|y_{i_{r}}-y_{i_{l}}\right|<0.25 \delta y$, we employ mesh movement and edge swapping to enforce $y_{i_{r}}=y_{i_{l}}[37,6]$, and if $\left|y_{i_{r}}-y_{i_{l}}\right| \geq 0.25 \delta y$ we introduce additional matching nodes on the opposite boundaries of the computational domain and reconnect them using supplementary edges. Alternatively, the later may be achieved by constructing for each unmatched periodic node, a degenerated polygonal finite-volume cell containing a hanging node(s) [37]. We found that both treatments perform similarly.

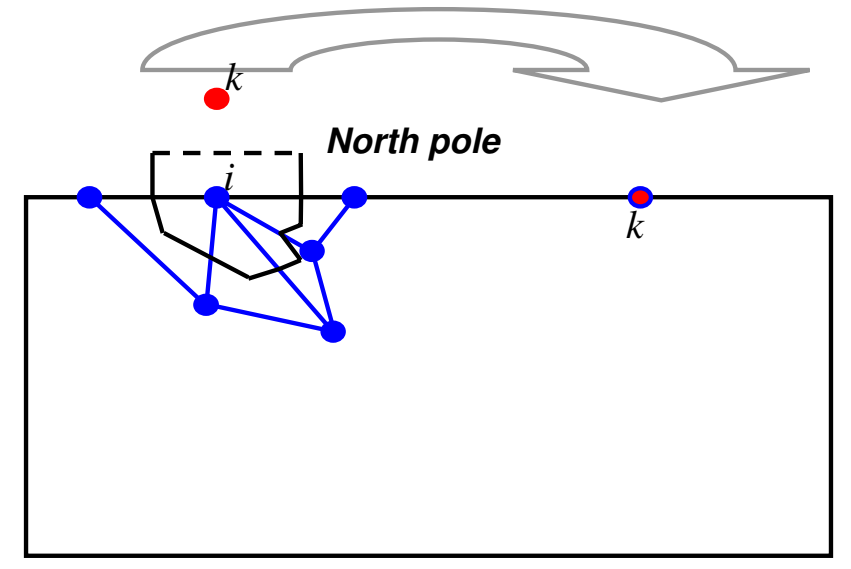

Fig. 3. A finite volume cell for a node neighbouring the north pole.

The polar boundary conditions are inherent in the geospherical framework. Here we describe the original formulation consistent with the unstructured finite-volume schemes outlined in sections 3.1 and 3.2. We avoid placing nodes at the poles, where the geospherical framework may introduce singularities in the archetype PDE (1) and the related discretisations. It is beneficial and easy, to generate a mesh such that the nodes closest to the pole are placed at the same distance from it. The distance is dictated by a half of the average internal edge length in this region. Figure 3 shows that these nodes form a solid line, parallel to the dashed line that corresponds to the position of the north pole. The resulting finite volume for a point $i$ is shown in the figure. The implementation of (14) for the point $i$ is the same as for any other node, because the normal velocity $V_{j}^{\perp}$ at the cell face (dashed line) on the pole is zero, hence there is no contribution from fluxes through this face. To substantiate, note that in (15) at the north pole cell face, the first term becomes zero because $\delta y=\pi / 2-\pi / 2=0$, and $h_{x}=r \cos (\pi / 2)$ nullifies the second term.

In the entire procedure (14-19) only steps (17) and (19) require special treatments in the polar regions. When evaluating derivatives (19) at the point $i$, the information from the node $k$ positioned on the sphere on the opposite side of the pole, is required to evaluate contributions form the cell face on the north pole. In the geospherical coordinates point $k$ (marked in red) corresponds to 
a mesh node placed at $x_{k}=x_{i}+\pi / 2$. This is schematically shown in Figure 3. During the unstructured mesh generation we ensure that for every node of the type $i$ a corresponding node $k$ exists. When the enforcement of the condition for the nodes $i$ and $k$ to be on the opposite sides of the pole impacts on mesh flexibility, we use values interpolated to the position $x_{i}+\pi / 2$. When computing the partial derivatives (17) that enter the antidiffusive velocities (16) of MPDATA, the contributions from the point $k$ are also required for evaluation of $\bar{\Psi}^{i, m} \equiv 0.5\left(\Psi_{i}+\Psi_{m}\right)$ whenever $m$ coincides with the point $k$. Then, for $\Psi$ s representing the momentum components, the contributions from $k$ are taken with an opposite sign. The treatment at the south pole follows the same principles.

\section{NUMERICAL RESULTS}

\subsection{Shallow-water equations on the Sphere}

The scalar advection of a cosine-bell summarised in table 1, tested the handling of kinematics. The two following benchmarks, a constant angular velocity zonal flow past an isolated mountain and the evolution of the Rossby-Haurwitz wave number 4 , test the handling of the planetary wave dynamics, epitomising global weather in linear and nonlinear regimes.

\subsubsection{Zonal orographic flow}

The first problem is a zonal flow past a conical hill centered in mid latitudes, studied by Grose and Hoskins [38]. The flow is characterised by small Rossby and Froude numbers (here $R_{o}=U / L f$ and $F r=U / \sqrt{g H}$, with $L$ denoting the horizontal scale of the problem) and, therefore, it is well explained by the linear theory [38]. This problem was proposed by Williamson et al. [33] for evaluating the efficacy of numerical methods for global-scale dynamics and has become a benchmark in the field. In [39] it was extended to 3D nonhydrostatic Boussinesq fluid, and employed to assess the relative merits of different analytic formulations of the governing equations versus truncation errors of different integration schemes. To allow the comparison with the results in [39] and, thus, to exemplify the capability of shallow-water equations to approximate longwave solutions of PDE systems governing 3D continuously stratified flows, here we follow the setup of [39]. The ambient zonal flow assumes $\mathbf{v}_{e}=\left(U_{0} \cos \varphi, 0\right)$, so the corresponding $H_{e}=H_{0}-0.5 g^{-1}\left(r f_{0}+U_{0}\right) U_{0} \sin ^{2} \varphi$ assures that the 
system (2) is satisfied identically in the absence of an orographic forcing. ${ }^{4}$ The hill embedded in the ambient flow is centered at $(\lambda, \varphi)=(3 \pi / 2, \pi / 6)$; it is $2 \cdot 10^{3} \mathrm{~m}$ high, and has the base radius $\pi / 9$. At the equator, the ambient wind $U_{0}=20 \mathrm{~ms}^{-1}$, whereas the depth of the fluid $H_{0}=8 \cdot 10^{3} \mathrm{~m}$.

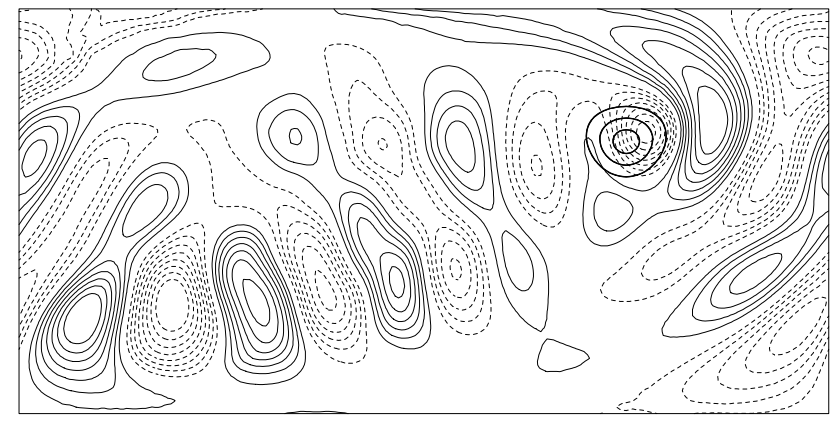

Fig. 4. Zonal orographic flow; meridional velocity component, after 15 simulated days, using an irregular triangular mesh with 8436 points. The contour interval is $2 \mathrm{~ms}^{-1}$ and no zero contour lines are shown. The hill contours are 0.25, 0.5 and 0.75 of its height.

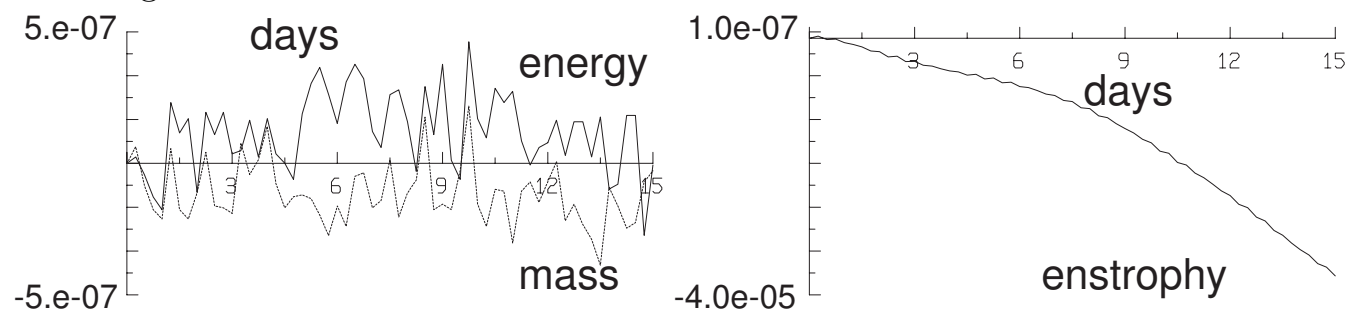

Fig. 5. Zonal orographic flow; mass, energy and potential enstrophy conservation errors, for single precision solution using an irregular triangular mesh.

Figure 4 shows the isolines of meridional velocity components after 15 (simulated) days. This solution was obtained on an irregular triangular mesh (of the type shown in Fig. 7) consisting of 8436 points, using the constant time step $\delta t=40 \mathrm{~s}$. The pattern is consistent with the linear solution of Grose and Hoskins (fig. 3 in [38]) and closely matches the Cartesian grid solution generated with EULAG for continuously stratified 3D nonhydrostatic Boussinesq fluid (Fig. 1 in [39]). Figure 5 complements the solution displayed in Fig. 4 with the history of the conservation errors. It shows the normalised (by the corresponding initial values) domain integrals of the mass, energy and potential enstrophy perturbations (with respect to the initial values) in function of time, sampled every simulated hour; the values obtained after 15 days are listed in table 2. The 15 day long conservation histories of mass and energy evince errors oscillating near numerical zero. For an equivalent double precision run (not shown) the mass conservation error drops to $\mathcal{O}\left(10^{-15}\right)$, whereas the energy and the potential enstrophy errors remain similar. This is unsurprising as, by design, the mass conservation is ensured to the round-off error;

4 Alternatively, the assumed ambient wind $\mathbf{v}_{e}$ is geostrophic, as it results from the balance of pressure and Coriolis forces. 
whereas the conservation of energy and enstrophy depends on truncation errors of the solution method as well as on the post-processing. Altogether, the results quoted appear to be consistent with corresponding results documented in the literature; cf. [40] for similar metrics obtained from the spectral-element calculations employing (presumably) 13 times more grid points.

Table 2

Zonal orographic flow; mass, energy and potential enstrophy conservation errors after 15 simulated days.

\begin{tabular}{cccc}
\hline \hline & mass & energy & potential enstrophy \\
single precision & $-2.8 \cdot 10^{-8}$ & $-6.1 \cdot 10^{-9}$ & $-3.7 \cdot 10^{-5}$ \\
double precision & $+3.9 \cdot 10^{-15}$ & $+2.3 \cdot 10^{-8}$ & $-3.7 \cdot 10^{-5}$ \\
\hline \hline
\end{tabular}

To further quantify the accuracy of the approach, table 3 summarises the mesh convergence study, for the solutions after 15 days. In the absence of a matching analytic solution, a numerical result obtained on a fine irregular triangular mesh, consisting of 121761 points, was taken as a reference. The error norms are calculated for a sequence of meshes with spatial resolution $\delta \xi$ decreasing as $\sqrt{2}$, with $\delta \xi_{k}=2^{-k / 2} \delta \xi_{0}, k=-1,0, . ., 3, \delta \xi_{0}=2.8125^{\circ}$, and the high-resolution reference mesh corresponding to $k=4$. The table shows ratios of the error norms $\left\|\psi_{k}^{\prime}\right\| /\left\|\psi_{k+1}^{\prime}\right\|$ (for height and velocity fields) in function of the ratio of consecutive mesh resolutions $\delta \xi_{k} / \delta \xi_{k+1}$ at a constant Courant number. Here, prime denotes deviation from the reference result mapped on a given mesh. The second-order asymptotic convergence rate is evident in the table.

Table 3

Zonal orographic flow on a sphere; the resolution ratios for consecutive meshes are shown together with the corresponding ratios of the solution error norms.

\begin{tabular}{ccccc}
\hline \hline$\delta \xi_{k} / \delta \xi_{k+1}$ & $2^{1 / 2} / 2^{0}$ & $2^{0} / 2^{-1 / 2}$ & $2^{-1 / 2} / 2^{-2 / 2}$ & $2^{-2 / 2} / 2^{-3 / 2}$ \\
$L_{1}\left(H_{k}^{\prime}\right) / L_{1}\left(H_{k+1}^{\prime}\right)$ & 1.75 & 1.87 & 1.88 & 2.00 \\
$L_{1}\left(v_{k}^{\prime}\right) / L_{1}\left(v_{k+1}^{\prime}\right)$ & 1.76 & 2.04 & 1.89 & 2.03 \\
$L_{2}\left(H_{k}^{\prime}\right) / L_{2}\left(H_{k+1}^{\prime}\right)$ & 1.93 & 1.92 & 1.90 & 1.98 \\
$L_{2}\left(v_{k}^{\prime}\right) / L_{2}\left(v_{k+1}^{\prime}\right)$ & 1.77 & 2.01 & 1.93 & 2.00 \\
\hline \hline
\end{tabular}

\subsubsection{Rossby-Haurwitz wave}

The Rossby-Haurwitz (RH) waves are analytic solutions of the solenoidal nonlinear barotropic vorticity equation on the sphere. They propagate zonally without change of shape [41], and are reminiscent of some large scale waves 


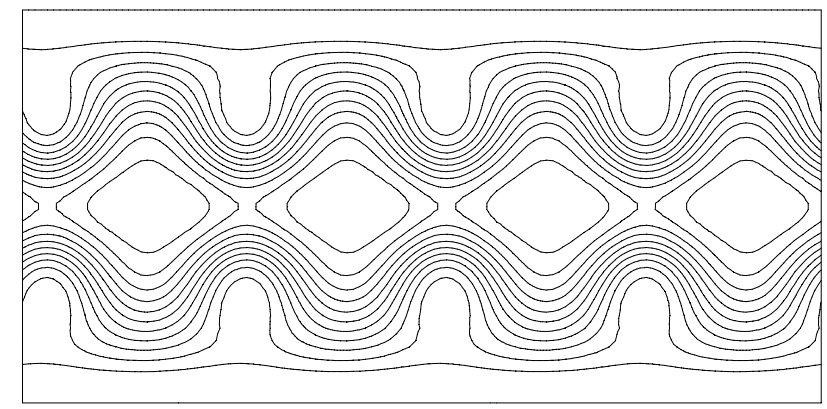

Fig. 6. Free-surface perturbation $H / H_{0}-1$ for the Rossby-Haurwitz wave number 4 test, after 5 simulated days; the contour interval is 0.025 .

in the atmosphere. However, the classical RH waves are not regularly observed in the atmosphere. Arguably, this is because they are unstable, given sufficiently short zonal wavelength and sufficient amplitude [42]. In particular, when simulated with the shallow water equations (thus admitting small perturbations to the analytic solutions with suppressed free surface) the wave with zonal wave number 4 was found to change its form a little over 24 days, while twice shorter RH waves break completely within a week [42]. Because of this marginal stability [43], $\mathrm{RH}$ wave number 4 is a convenient vehicle to test capability of numerical methods for maintaining subtle nonlinear balance of wave form solutions over extended integration time. The simulation of the RH wave number 4 with shallow water equations was included in the suite of tests proposed by Williamson et al. [33], and has become another benchmark in the field. Figure 6 shows our solution after 5 days of the simulated time, obtained on an irregular triangular mesh consisting of 7722 points. This result is in good agreement with theoretical estimates $(0.34 \pi$ eastward displacement after 5 days), and it reproduces the Cartesian-grid solution documented in $[34]$.

The edge-based structure of the flow solver permits its application on arbitrary polygonal meshes. To examine sensitivity of the solutions to details of the mesh, the test was repeated using Cartesian and triangularised Cartesian meshes (8256 points) as well as an equilateral triangular mesh with 8352 points operating on equilateral hexagonal finite volumes; Fig. 7. The corresponding solutions are not shown because their departures from the results in Fig. 6 are insignificant, despite unavoidable small differences in mesh resolution. The solution in Fig. 8 uses a distinct mesh. It was obtained on a mesh with only 5764 points distributed irregularly in the computational space, but with a near equidistribution of points in the physical space. This is illustrated in Fig. 9 that shows an akin mesh, coarsened for visual clarity. Some departures from symmetry in the polar regions seen in Fig. 8 indicate a need for more than 8 points in a polar circumference to avoid overstretched finite volumes in the geospherical framework and to resolve fields' gradients. This is consistent with earlier findings using reduced grids in high latitudes; see [1] 


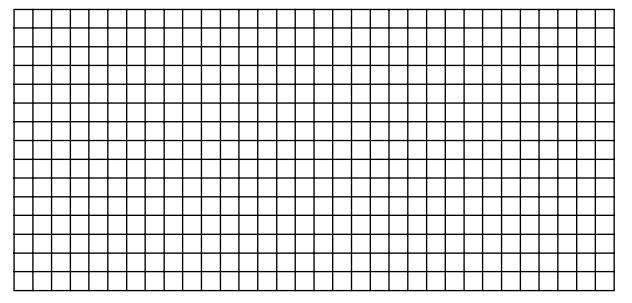

Cartesian

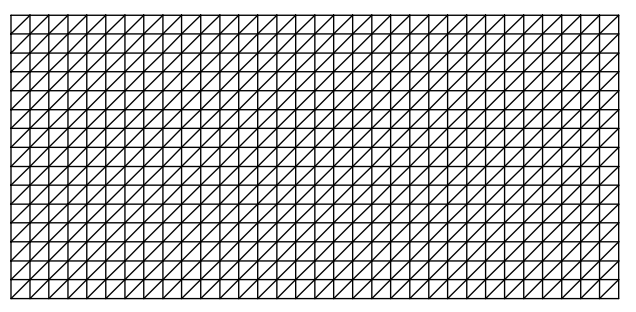

triangularized Cartesian

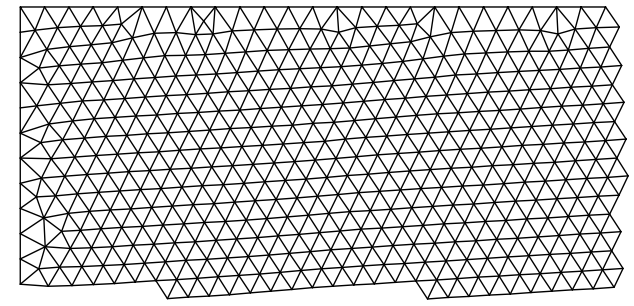

irregular triangular

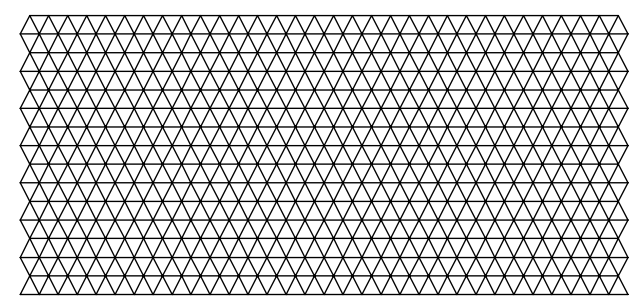

equilateral triangular

Fig. 7. Selection of computational meshes (zoomed on the upper left quarters) used by the edge-based solver for the Rossby-Haurwitz wave benchmark.

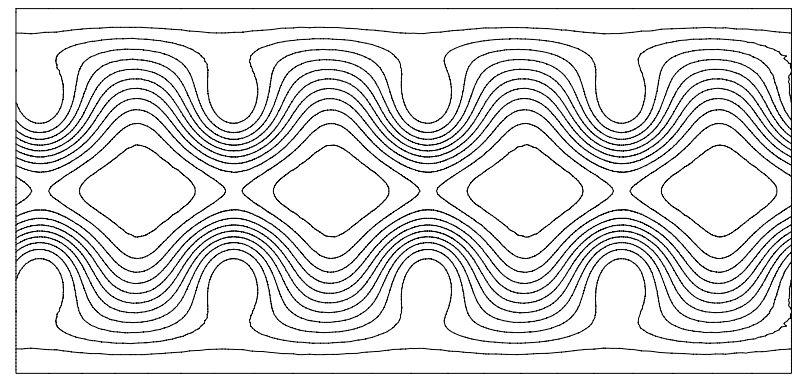

Fig. 8. As in Fig. 6, but for the free-surface perturbation obtained on the mesh equidistributed in the physical space on a sphere

and references therein.
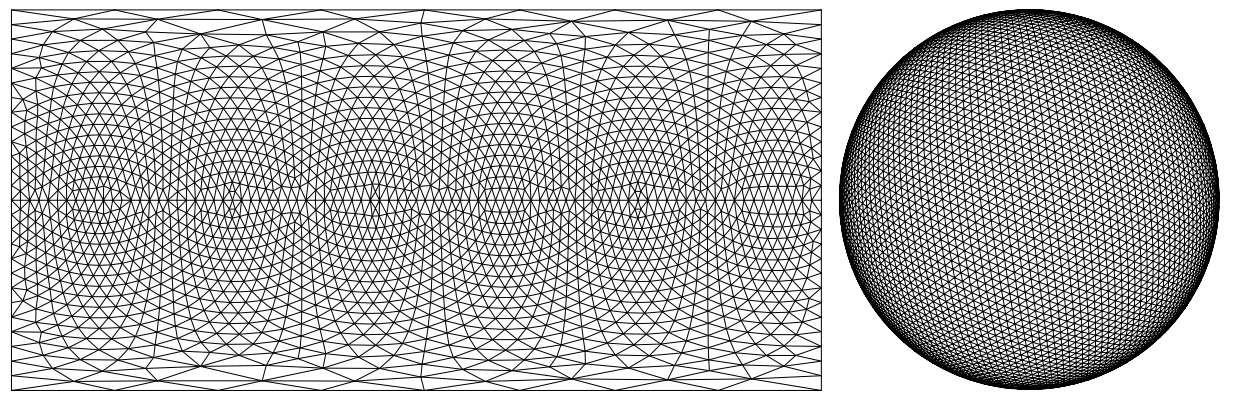

Fig. 9. Evenly distributed mesh in the physical space on a sphere

All tests were continued to verify the solutions after 14 days. Almost indistinguishable results were obtained on the Cartesian and equilateral triangular 
meshes, Fig. 10, and they are consistent with predictions of a spectral model [40]. However, the solution obtained on the triangularised Cartesian mesh, Fig. 11, shows a pronounced North-South asymmetry. This mesh was purposely designed to assess an influence of anisotropic meshing on simulated wave propagation. Notably, a similar experiment with the same triangularised Cartesian mesh, but with antisymmetric slopes of hypotenuses across the equator does recover the solution symmetry (not shown).

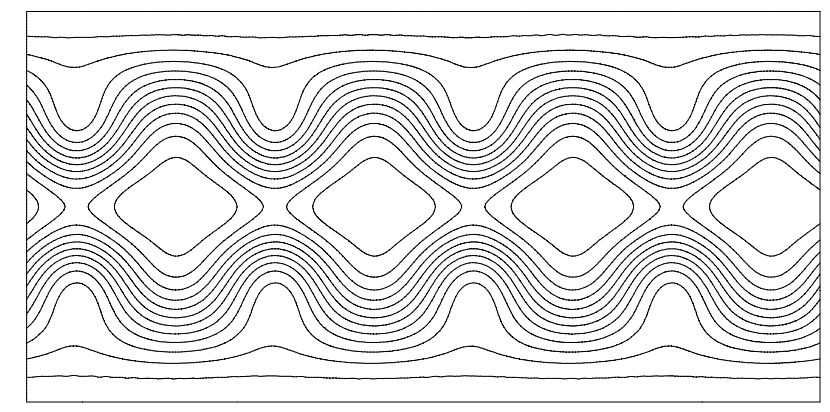

Fig. 10. As in Fig. 6, but for the solution after 14 days, using the equilateral triangular mesh.

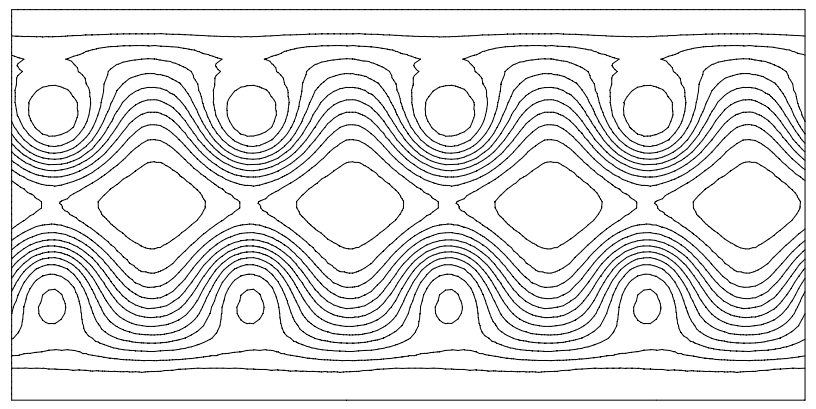

Fig. 11. As in Fig. 10, but using the directionally-biased triangularised Cartesian mesh

The solution obtained on the irregular triangular mesh with 7722 points, Fig. 12, indicates unstable waves. A stable wave solution, close to that shown in Fig. 10 was regained for an irregular triangular mesh containing 30589 points; i.e., with a halved resolution. Further investigation showed this test case to favor meshes, the truncation errors of which do not interfere with the solution symmetry. In particular, for regular grids (especially for Cartesian) the wave solutions remained stable for long simulation times. However, even small perturbations triggered by mesh truncation errors ultimately led to growth of some unstable modes. The manifestation of influence of such perturbations occurred at different simulated lengths of time and depended on the mesh resolution and the integration time step (viz. Courant number). A similar unstable wave behaviour resulted from a test using the Cartesiangrid EULAG-type solver employed in [34], but with a small random noise 
introduced in the initial condition. These observations are compatible with findings of the study reported in [43] that, contrary to common beliefs, the zonal wavenumber 4 Rossby-Haurwitz wave is dynamically unstable and will eventually break if sufficiently perturbed.

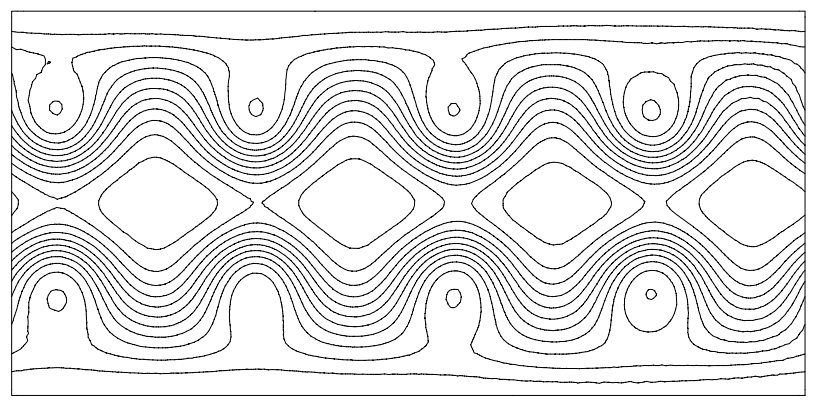

Fig. 12. As in Fig. 10, but for unstable solution on the coarser (7722 points) irregular triangular mesh.

Figure 13 documents that the wave instability in the solution highlighted in Fig. 12 has no distinct impact on the conservation of mass, energy and enstrophy. At day 14 , the respective single-precision error values are $3.85 \cdot 10^{-7}$, $-1.36 \cdot 10^{-5}$ and $-1.91 \cdot 10^{-4}$. From the considered integral error measures, only the unrealistically high value of the $L_{2}$ norm ratio in Fig. 14 recorded at day 11 evidences the emergence of fine scales in the $\mathrm{RH}$ wave pattern on the coarse mesh, indicative of the growing instability. The $L_{2}$ height error was computed by taking a solution on a fine Cartesian mesh (1025x505) as a reference. The slope in figure 14 represents a ratio between $L_{2}$ norms calculated using triangular meshes consisting of 7722 and 30589 points.

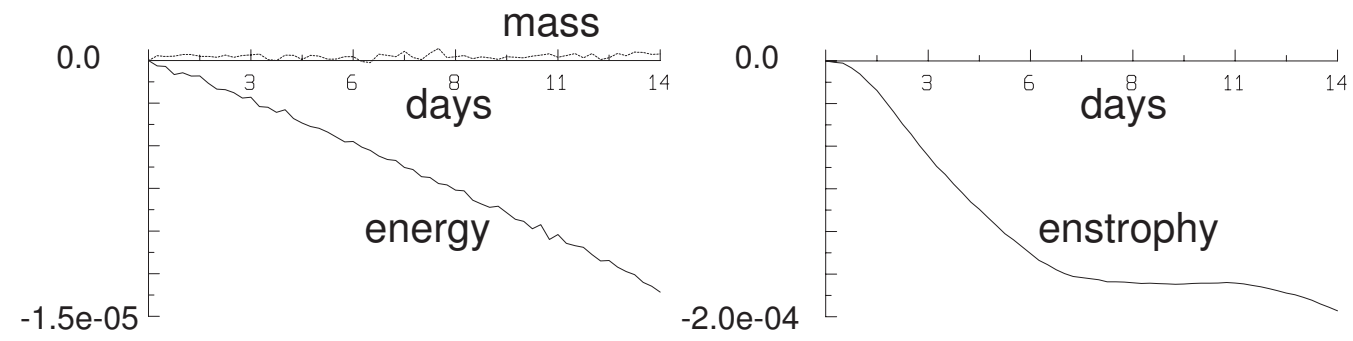

Fig. 13. Mass, energy and potential enstrophy conservation errors, in single precision, a solution of the Rossby-Haurwitz wave benchmark, using an irregular 7722 points triangular mesh

\subsection{D extension}

Examples of the preceding section illustrate the performance of the proposed approach, in the context of smooth large-scale motions excited with weak perturbations from equilibrated reference states. Here, we consider a stiff problem 


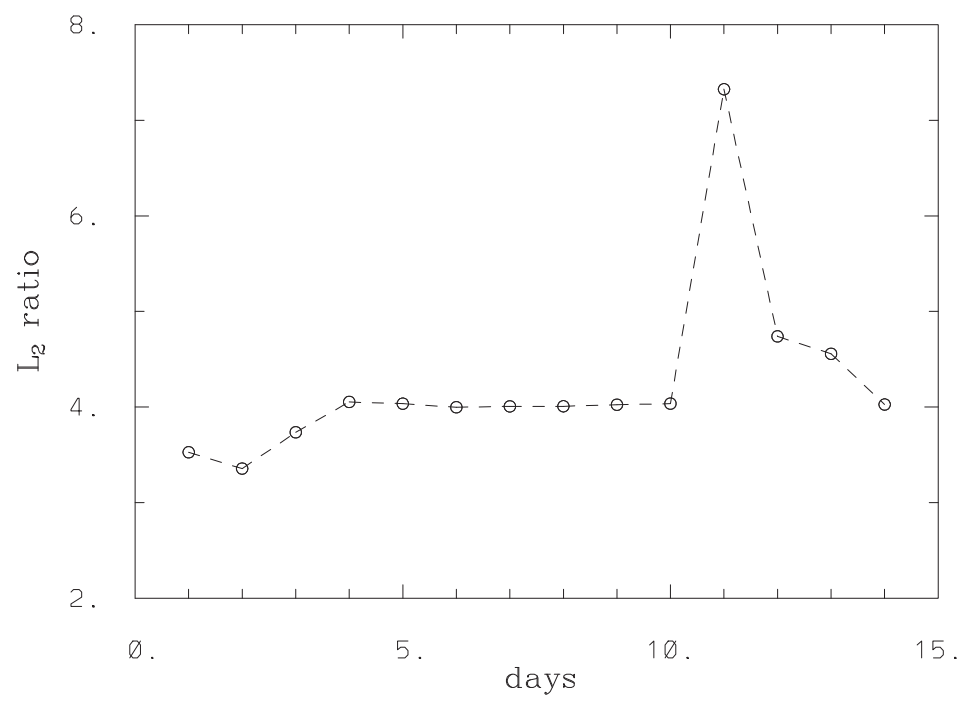

Fig. 14. A history of the $L_{2}$ height error norm ratio between a coarse and a twice finer irregular triangular meshes. The Rossby-Haurwitz wave test problem

with multiplicity of emerging solution scales. For this purpose, we employ 3D primitive equations expressed in a hybrid Eulerian-Lagrangian form with a material coordinate $\zeta$ monotonically increasing with height, cf. section 7.4 in [21]. Such formulation is close in form to a coupled set of shallow water layers (2). The governing PDEs can be written compactly as

$$
\begin{gathered}
\frac{\partial G \mathcal{D}}{\partial t}+\nabla \cdot\left(G \mathbf{v}^{*} \mathcal{D}\right)=0 \\
\frac{\partial G Q_{x}}{\partial t}+\nabla \cdot\left(G \mathbf{v}^{*} Q_{x}\right)=G\left(-\frac{1}{h_{x}} \mathcal{D} \frac{\partial M}{\partial x}+f Q_{y}-\frac{1}{G \mathcal{D}} \frac{\partial h_{x}}{\partial y} Q_{x} Q_{y}\right) \\
\frac{\partial G Q_{y}}{\partial t}+\nabla \cdot\left(G \mathbf{v}^{*} Q_{y}\right)=G\left(-\frac{1}{h_{y}} \mathcal{D} \frac{\partial M}{\partial y}-f Q_{x}+\frac{1}{G \mathcal{D}} \frac{\partial h_{x}}{\partial y} Q_{x}^{2}\right) \\
\frac{\partial M}{\partial \zeta}=\Pi
\end{gathered}
$$

where, in contrast to $(2)$, the position vector $\mathbf{x}=(x, y, \zeta)$ has now three components, and the generalised density is $\mathcal{D} \equiv \partial p / \partial \zeta$. The pressure gradient force depends on the Montgomery potential (or Bernoulli function) $M=g H+$ $\zeta \Pi$, where $H$ is the height of a material surface, and $\Pi$ is a transform of the pressure $p$. Consequently, the last equation in (21) is an expression of the hydrostatic balance. For example in isentropic model [45] $\zeta \equiv \theta$, where $\theta$ is the potential temperature, so $\Pi$ is the Exner function; ${ }^{5}$ whereas in the isosteric/isopycnic model $[46,47], \zeta=\rho^{-1}$ is the specific volume, so $\Pi \equiv p$. Furthermore, to the Boussinesq approximation the isopycnic model amounts

5 The Exner function is $\Pi=c_{p}\left(p / p_{o}\right)^{R_{d} / C_{p}}$, where $c_{p}$ is the specific heat at constant pressure, $R_{d}$ is the gas constant for dry air, and $p_{o}$ is a reference pressure. 
to the isentropic one; cf. [46].

All prognostic equations of the system (21) adhere to the form of an archetype PDE (1). Their integration follows the template algorithm described in section 3.1, where all outlined steps apply now simultaneously to all discrete values of $\zeta$. The only significant difference between integrating the shallow water equations (2) and (21) lies in the recovery of pressure. In the case of shallow water, the pressure gradient force is readily computable after integrating the mass continuity equation with the homogeneous form of (7). In the case of (21), it involves the additional step of integrating the tridiagonal problem implied by the hydrostatic-balance relation. First, assuming the free-surface boundary condition aloft, the pressure $p$ is recovered by integrating $\mathcal{D}^{n+1}=\partial p^{n+1} / \partial \zeta$ downward, with successive upward integration of $\partial M^{n+1} / \partial \zeta=\Pi^{n+1}$, to establish the values of $M^{n+1}$ for all discrete values of $\zeta$. Subsequently, the momenta are evaluated for all $\zeta$ following the procedure outlined in section 3.1. All details of the discretisation in the horizontal follow the description in sections 3.2 and 3.3.

The physical problem addressed in this section is a response of stratified fluid to mesoscale orographic forcing at a range of Froude numbers; here $F r=U_{0} / N h_{0}$, with $U_{0}, N$ and $h_{0}$ denoting, respectively, characteristic values of ambient wind, buoyancy frequency, and mountain height. To simulate atmospheric mesoscale motions without incurring the computational expense associated with discretising the Earth surface with mesoscale resolutions [44,14], we reduce the planet's radius hundredfold; see [32] for a thorough exposition.

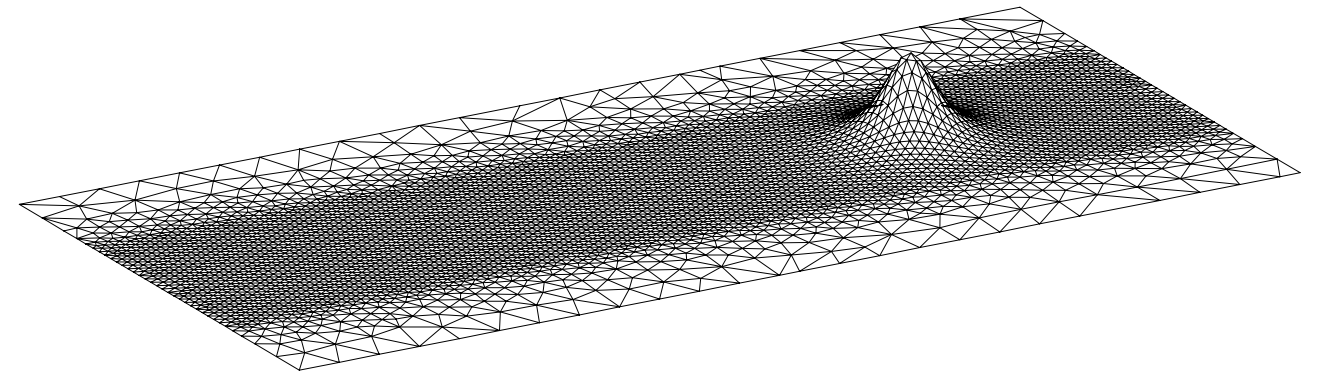

Fig. 15. Perspective display of the mesh (4532 points) used in 3D orographic-flow experiments; the vertical scale is exaggerated for clarity of visualisation.

The experimental setups are as follows. The ambient wind $\mathbf{v}_{e}=\left(U_{0} \cos \varphi, 0\right)$, with $U_{0}=10 \mathrm{~ms}^{-1}$. Following section 4.1.1, the corresponding undisturbed height of the material surfaces is specified as $H_{e}\left(x, y, \zeta_{k}\right)=(k-1) \delta z-$ $0.5 g^{-1}\left(r f_{0}+U_{0}\right) U_{0} \sin ^{2} \varphi$, with uniform separation in the vertical $\delta z=40 \mathrm{~m}$ and $k=1, \ldots, 91$. The assumed buoyancy frequency $N=0.04 \mathrm{~s}^{-1}$ defines the vertical scale of the problem in terms of the wavelength $\lambda_{Z}=2 \pi U_{0} / N=$ $1571 \mathrm{~m}$ of the dominant vertically propagating hydrostatic mountain wave. In order to relate to linear solutions in [46], the analytic axisymmetric hill is placed at the equator; cf. [32] for a discussion. Its height decays as $h(l)=$ 
$h_{0}\left[1+(l / \mathcal{L})^{2}\right]^{-3 / 2}$, where $l(x, y)$ denotes the distance from the centre of the hill on the spherical surface, and $\mathcal{L}=12.4 \cdot 10^{3} \mathrm{~m}$ is the hill's profile halfwidth. The calculations conducted for $h_{0}=125,250,500 \mathrm{~m}$, correspond to $F r=2,1,0.5$. Figure 15 illustrates the surface topography $\left(h_{0}=500 \mathrm{~m}\right)$ and the horizontal mesh consisting of 4532 points. The coarsest resolution is $12.3^{\circ}$, in the polar regions. In the equatorial region and the vicinity of significant topography the mesh is refined, with the resolution of $2.8^{\circ}$.

Calculations were carried for $T=4 \mathrm{~h}$ of the simulated time, starting with the ambient flow perturbed impulsively by the presence of the hill, such that $\left.H(\mathbf{x}, 0)=\max \left[H_{e}(\mathbf{x}), h(\mathbf{x}), \varepsilon\right]\right)$. Notably, this introduces material layers with vanishing thickness at the mountain slopes. Thanks to the positivity of the NFT numerics this poses no technical difficulties [47]. The integration time step $\delta t=8 \mathrm{~s}$ is limited by the CFL condition accounting for the phase speed of the external gravity wave $\left(\approx 200 \mathrm{~ms}^{-1}\right)$. The total simulated time is $T \approx 2 \tau_{g w} \approx 12 \tau_{a d v}$, where the gravity-wave time scale $\tau_{g w}=\lambda_{z} / c_{g z}$ assumes a linear estimate of the group velocity in the vertical $c_{g z}=U_{0}^{2} / \mathcal{L} N$, and $\tau_{a d v}=\mathcal{L} / U_{0}$ is an advective time scale. Noteworthy, by comparison with the standard Cartesian 128x64 grid with the resolution $2.8^{\circ}$, the unstructured mesh discussed has the same resolution on the equator and is about four times coarser near the poles, whereas the employed $\delta t$ is eight times larger than the corresponding time step permissible in the runs performed with the Cartesian grid (not shown).

The developed solutions for three selected Froude numbers $(F r=2,1$ and 0.5$)$ are highlighted in Figs. 16 and 17; to facilitate the visualisation, the results were interpolated to a fine Cartesian grid in the horizontal. The heights of isopycnals are shown after 4 hours of simulated time in the vertical plane at the equator, and along the undisturbed surface $H_{e}$ with maximum equatorial elevation at about the eighth of the vertical wavelength. ${ }^{6}$ The three results shown capture (from the top down) the transition from the linear regime at $F r \gtrsim 2$ to a fully nonlinear flow at $F r \lesssim 0.5$ [48]. At $F r=2$ the flow is predominantly over the hill, with all isopycnals gently displaced by the hill height - in a qualitative agreement with the predictions of the infinitesimalamplitude linear theory [46]. At $F r=1$, isopycnals collapse at the lee slope of the hill, and the tendency is apparent for the stagnation near the hill top on the windward side, concomitant with the steepening of the isopycnals aloft on the lee - still consistent with the linear theory predictions [46]. At $\mathrm{Fr}=0.5$, the flow below the dimensionless height $z / h_{0}=1-F r$ is predominantly around the hill. It is blocked at the windward side, as evidenced by isopycnals ending at the hill and by a large deflection of the flow vectors in the horizontal. In

6 Because of the shallowness of the domain, in the problem posed, the corresponding calculations with the isentropic model produce similar results, with only slightly larger wave amplitude aloft. 


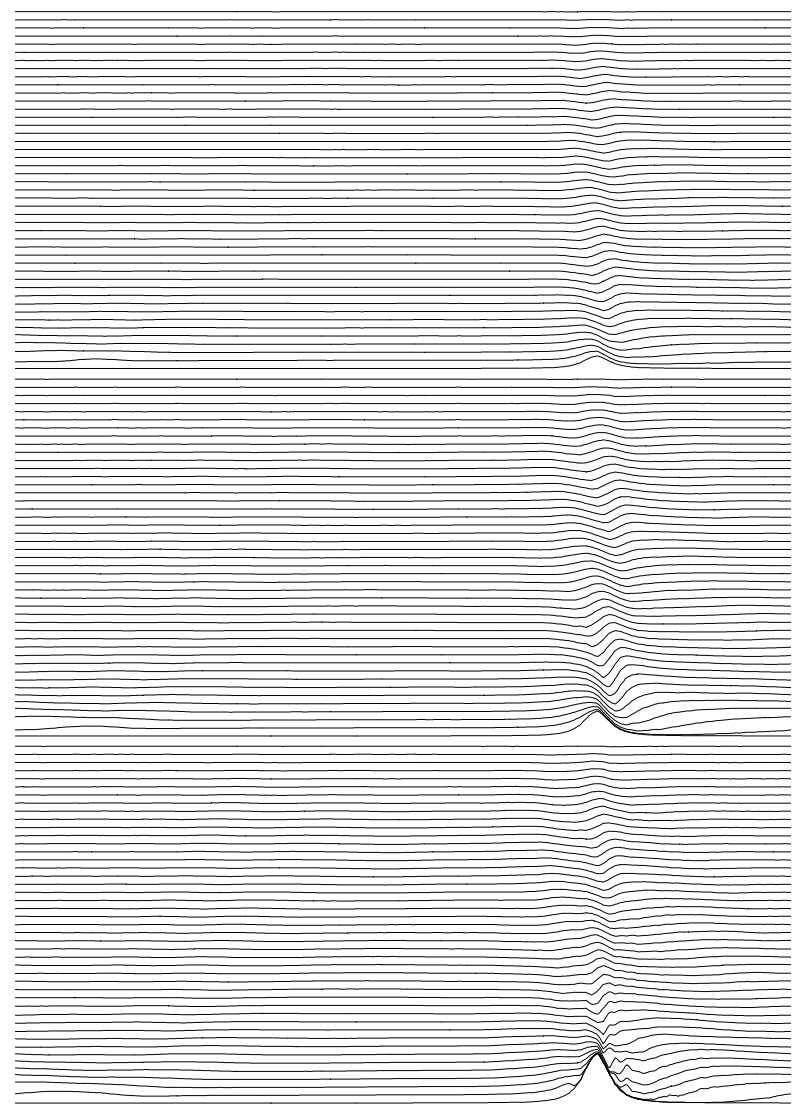

Fig. 16. Isopycnal elevation after $4 \mathrm{~h}$ of simulated time in the vertical equatorial cross section; $F r=2,1$ and 0.5 from the top to bottom.

the lee, the flow evinces complex topology indicative of the intense low-level eddies with the reversed uphill flow $[49,48]$; see [50] for a succinct review of this intricate problem.

\section{REMARKS}

A concept was proposed and explored of using unstructured meshes for discretising PDEs of geophysical fluid dynamics cast in the classical anholonomic latitude-longitude (lat-lon) spherical framework (viz. the geospherical framework). The results demonstrate that multiple benefits of the geospherical coordinates extensively exploited in the standard lat-lon finite difference and spectral models hold in the context of unstructured meshes. This first (to our knowledge) generalisation of the geospherical framework to fully unstructured meshes illustrated here with finite volume discretisation (viz linear elements) indicates the suitability of the concept for arbitrarily shaped hybrid meshes which can include higher order elements/discretisations.

The approach adds flexibility in terms of mesh anisotropy and inhomogene- 

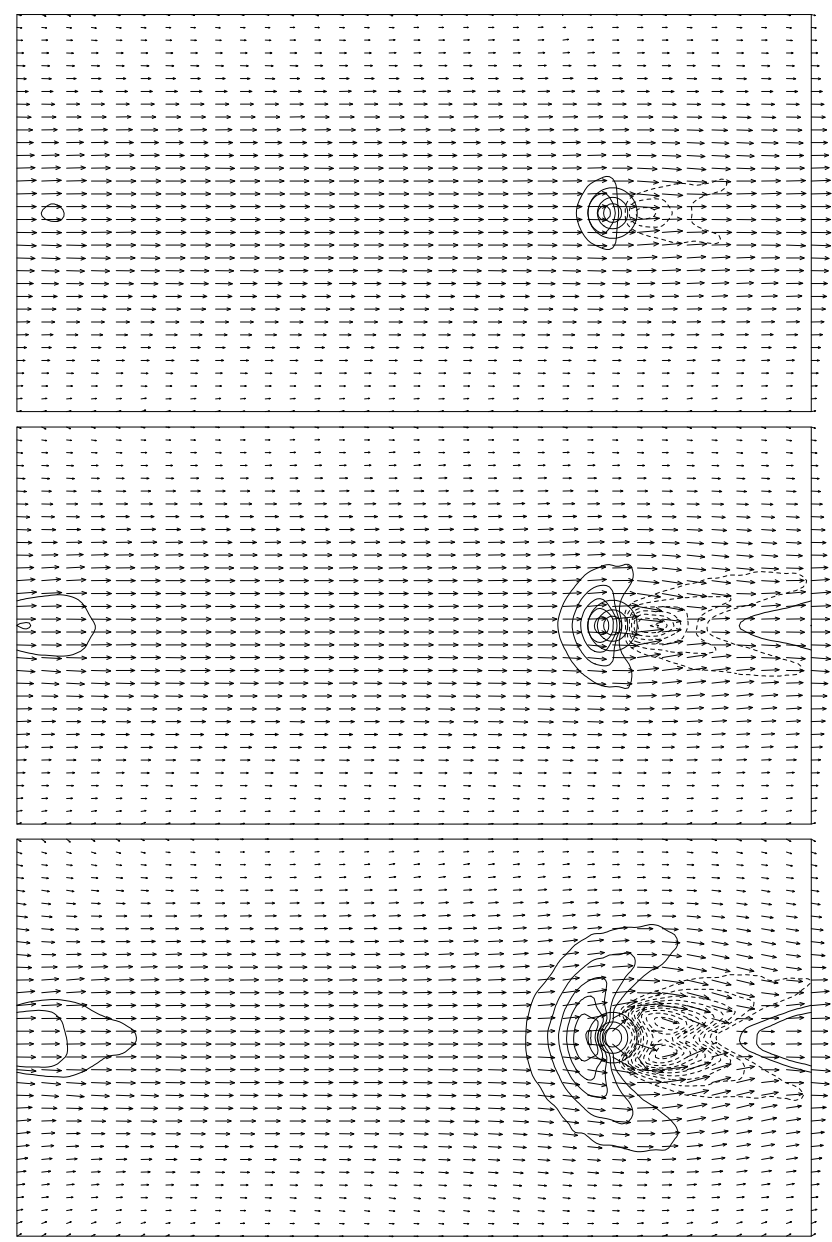

Fig. 17. Displacement field of the isopycnal surfaces with the equatorial undisturbed height $H_{e} \approx \lambda_{z} / 8$, for $F r=2,1$ and 0.5 , correspondingly to Fig. 16; contour interval is $\approx 0.01 \lambda_{z}$, no zero contour lines are shown. The reference arrow length for the superimposed flow is $U_{0}$ far upstream of the hill centre.

ity unattainable by many discretisation schemes. This is important for the efficacy of discretisation and for mesh adaptivity. A broad variety of computational meshes can be easily implemented as shown in examples of mesh with local static refinement used in the three-dimensional computations and a study of sensitivity of the solutions to details of computational mesh in two-dimensions. In principle, the proposed concept admits as special cases all former discretisations which used geospherical framework.

The presented generalisation of EULAG numerics to unstructured meshes retains proven properties of NFT MPDATA structured grid solvers. Indeed, the proposed framework promotes an implementation/translation of mature structured-mesh based methodologies building on a wealth of existing experience. Higher resolutions, necessary to fully resolve detailed flow features in highly multiscale geophysical flows are not available in current structured meshes based global models. Flexible unstructured and adaptive meshes may 
offer an efficient alternative to the many-fold increase in computational points required by lat-lon grids to achieve such desired resolutions. The proposed framework is suitable for studying the potential of unstructured meshes in this context.

Acknowledgments: Comments from Joseph Prusa and an anonymous referee helped to improve the presentation. This work was supported in part by the DOE award DE-FG02-08ER64535 and the NERC award NE/G004358. The National Center for Atmospheric Research is sponsored by the National Science Foundation.

\section{References}

[1] D.L. Williamson, The Evolution of Dynamical Cores for Global Atmospheric Models, Journal of the Meteorological Society of Japan, 85B (2008) 241-269

[2] M. Lauter, D. Handorf, N. Rakowsky, J. Behrens, S. Frickenhaus, M. Best K. Dethloff, W. Hiller, A parallel adaptive barotropic model of the atmosphere, J. Comput. Phys., 223 (2007) 609-628.

[3] A. Evans, M.J. Marchant, J. Szmelter, N.P. Weatherill, Adaptivity for Compressible Flow Computations using Point Embedding on 2-D structured Multiblock Meshes, Int. J. Numer. Methods Eng., 32 (1991) 896-919.

[4] N.P. Weatherill, Grid generation, Von Karman Lecture Notes, June 1989. 1-96, ISSN 0377-8312.

[5] J.F. Thompson, B.K. Soni, N.P. Weatherill, Handbook of Grid Generation, CRC Press, ISBN 0-8493-2687-7, (1999).

[6] J. Szmelter, P.K. Smolarkiewicz, MPDATA Error Estimator for Mesh Adaptivity, Int. J. Numer. Methods Fluids 50 (2006) 1269-1293.

[7] J. Szmelter, A. Pagano, Viscous Flow Modelling using Unstructured Meshes for Aeronautical Applications, Lecture Notes Physics 453 (1995) 293-298.

[8] J. Wu, J.Z. Zhu, J. Szmelter, O.C. Zienkiewicz, Error Estimation and Adaptivity in Navier-Stokes Incompressible Flows, Comp. Mech. 6 (1990) 259270

[9] P. Ortiz P, O.C. Zienkiewicz, J. Szmelter, Hydrodynamics and Transport in Estuaries and Rivers by the CBS Finite Element Method, Int. J. Num. Meth. Eng. 66 (2006) 1569-1586.

[10] R. Sadourny, A. Arakawa, Y. Mintz, Integration of the Nondivergent Barotropic Vorticity Equation with an Icosahedral-Hexagonal Grid for the Sphere, Monthly Weather Rev., 96 (1968) 351-356. 
[11] D. Williamson, Integration of the Barotropic Vorticity Equation on a Spherical Geodesic Grid, Tellus, 20 (1968) 642-653.

[12] R. Sadourny, Conservative Finite-Difference Approximations od the Primitive Equations on Quasi-Uniform Spherical Grids, Monthly Weather Rev., 100 (1972) 136-144.

[13] D. Williamson, Review of Numerical Approaches for Modelling Global Transport, Air Pollution Modeling and its Application IX, Edited by H. von Dop and G. Kallos Plenum Press, New York, (1992) 377-394.

[14] M. Satoh, T. Matsuno, H. Tomita, H. Miura, T. Nasuno, S. Iga: Nonhydrostatic icosahedral atmospheric model (NICAM), J. Comput. Phys. 227 (2008) 34863514 .

[15] A. Staniforth, N. Wood, Aspects of the dynamical core of a nonhydrostatic deep-atmosphere, unified weather and climate-prediction model. J. Comput. Phys. 227 (2008) 3445-3464.

[16] M. Lauter, F.X. Giraldo, D. Handorf and K. Dethloff, A discontinuous Galerkin method for the shallow water equations in spherical triangular coordinates, J. Comput. Phys. 227 (2008) 10226-10242.

[17] S. Li, F. Xiao, A global shallow water model using high order multi-moment constrained finite volume methods and icosahedral grid, J. Comput. Phys. 229 (2010) 1774-1796.

[18] J. Behrens, Adaptive atmospheric modelling: key techniques in grid generation, data structures, and numerical operations with applications, Lecture notes in Computational Science and Engineering, Springer (2006).

[19] D.P. Bacon et al., A dynamically adapting weather and dispersion model: the operational environment model with grid adaptivity (OMEGA), Monthly Weather Rev. 128 (2000) 2044-2076.

[20] Maurin, K., 1980: Analysis Part II: Integration, Distributions, Holomorphic Functions, Tensor and Harmonic Analysis, Reidel Publishing Co., Boston, 829 pp.

[21] Dutton JA. The Ceaseless Wind. Dover Publications, 1986, pp. 617.

[22] E.F. Toro, Riemann Solvers and Numerical Methods for Fluid Dynamics, 2nd Edition, Springer, 1999, 624 pp.

[23] P.E. Bernard, J.F. Remacle, R. Comblen, V. Legat, K. Hillewaert, High-order discontinuous Galerkin schemes on general 2D manifolds applied to the shallow water equations, J. Comput. Phys. 228 (2009) 6514-6535.

[24] J.M. Prusa, P.K. Smolarkiewicz, A.A Wyszogrodzki, EULAG, a Computational Model for Multiscale Flows, Comput. Fluids 37 (2008) 1193-1207.

[25] J.M. Prusa, P.K. Smolarkiewicz, An all-scale anelastic model for geophysical flows: dynamic grid deformation, J. Comput. Phys. 190 (2003) 601-622. 
[26] P.K. Smolarkiewicz, J.M. Prusa, Toward mesh adaptivity for geophysical turbulence: continuous mapping approach. Int. J. Num. Methods Fluids 47 (2005) 789-801.

[27] P.K. Smolarkiewicz, Multidimensional positive definite advection transport algorithm: an overview, Int. J. Numer. Methods Fluids, 50 (2006) 1123-1144.

[28] P.K. Smolarkiewicz, J. Szmelter, MPDATA: An Edge-Based Unstructured-Grid Formulation, J. Comput. Phys. 206 (2005) 624-649

[29] P.K. Smolarkiewicz, J. Szmelter, Multidimensional positive definite advection transport algorithm (MPDATA): An edge-based unstructured-data formulation. Int. J. Numer. Meth. Fluids, 47, (2005), 1293-1299.

[30] P.K. Smolarkiewicz, J. Szmelter, An MPDATA based solver for compressible flows, Int. J. Numer. Methods Fluids 56 (2008) 1529-1534.

[31] P.K. Smolarkiewicz, J. Szmelter, Iterated Upwind Schemes for Gas Dynamics, J. Comput. Phys, 228 (2009) 33-54.

[32] N.P. Wedi, P.K. Smolarkiewicz, 2009: A Framework for testing global nonhydrostatic models. Q.J. Roy. Meteorol. Soc., 135 (2009) 469-484.

[33] D.L. Williamson, J.B. Drake, J.J. Hack, R. Jakob, P.N. Swarztrauber, A Standard Test Set for Numerical Approximations to the Shallow Water Equations in Spherical Geometry, J. Comput. Phys. 102 (1992) 211-224.

[34] P.K. Smolarkiewicz, L.G. Margolin, MPDATA: A finite-difference solver for geophysical flows, J. Comput. Phys. 140 (1998) 459-480.

[35] P.K. Smolarkiewicz, L.G. Margolin, On forward-in-time differencing for fluids: extension to a curvilinear framework, Monthly Weather Rev. 121 (1993) 18471859 .

[36] J.A. Pudykiewicz, Numerical Solution of the Reaction-Advection-Diffusion Equation on the Sphere, J. Comput. Phys. 213 (2006) 358-390.

[37] J. Szmelter, M.J. Marchant, A. Evans, N.P. Weatherill, Two-dimensional Navier-Stokes equations - adaptivity on structured meshes, Computer Methods in Applied Mechanics and Engineering, 101 (1992) 355-368.

[38] W.L. Grose and B.J. Hoskins, On the influence of orography on large-scale atmospheric flow, J. Atmos. Sci, 36 (1979), 223-234.

[39] P.K. Smolarkiewicz, L.G. Margolin and A.A. Wyszogrodzki, A Class of Nonhydrostatic Global Models, J. Atmos. Sci, 58 (2001), 349-364.

[40] M. Taylor, J. Tribbia and M. Iskandarani, The spectral element method for the shallow water equations on the sphere, J. Comput. Phys. 130 (1997) 92-108.

[41] B. Haurwitz, The motion of atmospheric disturbances on the spherical Earth, J. Marine Res., 3 (1940) 254-267.

[42] B.J. Hoskins, Stability of the Rossby-Haurwitz wave, Q.J. Roy. Meteorol. Soc., 99 (1973) 723-745. 
[43] J. Thuburn and Y. Li, Numerical simulations of Rossby-Haurwitz waves, Tellus Series A-dynamic Meteorology and Oceanography 52 (2000) 181-189.

[44] B.W. Shen, R. Atlas, J.D. Chern, O. Reale, S.J. Lin, T. Lee and J. Chang, The 0.125 degree finite-volume general circulation model on the NASA Columbia supercomputer: Preliminary simulations of mesoscale vortices, Geophys. Res. Lett., 33 (2006) L05801.

[45] J.M. Reisner and P.K. Smolarkiewicz, Thermally forced low Froude number flow past three-dimensional obstacles, 51, J. Atmos. Sci., (1994) 117-133.

[46] R.B. Smith, Linear theory of hydrostatic flow over an isolated mountain in isosteric coordinates, 45, J. Atmos. Sci., (1988) 3887-3896.

[47] C. Schär, P.K. Smolarkiewicz, A synchronous and iterative flux-correction formalism for coupled transport equations, 128, J. Comput. Phys. (1996) 101120 .

[48] P.K. Smolarkiewicz, R. Rotunno, Low Froude number flow past threedimensional obstacles. Part I: Baroclinically generated lee vortices, J. Atmos. Sci. 46 (1989), 1154-1164.

[49] C.R. Hunt, W.H. Snyder, Experiments on stably and neutrally stratified flow over a model three-dimensional hill, J. Fluid Mech. 96 (1980) 671-704.

[50] C.C. Epifanio, R. Rotunno, The dynamics of orographic wake formation in flows with upstream blocking, J. Atmos. Sci. 62 (2005) 3127-3150. 